\title{
Transformation of Ladder Based All Pass and Biquad Filters into Active All Pass and Biquad Filter using CFOA
}

\section{Praween K Sinha* and Neelam Sharma}

Electronics and Communication Department, Maharaja Agrasen Institute of Technology, New Delhi, India

\begin{abstract}
In this paper ladder based all pass and biquad filters are transformed into CFOA based all pass and biquad filters. The ladder based approach is used for design of prototypes. The new circuits have attractive properties of greater linearity, high dynamic rate, high slew rate and high signal bandwidth. A new technique for the conversion has been proposed. The technique uses signal flow graph and converts the existing LC ladder based filter into CFOA based configurations. The design of all pass and Biquad filter has been realized using the proposed technique. The proposed configuration is implemented using CFOA as an active device and all the capacitors are grounded. The new configurations are suitable for various signal processing applications. Simulation has been carried out using simulation software PSpice (v10.2). The simulation results have been demonstrated and discussed.
\end{abstract}

Keywords: Current feedback operational amplifier; Ladder filter; Signal flow graph; Current mode; Voltage mode; All pass filter; Biquad filter

\section{Introduction}

Signal flow graph is a diagram that represents set of simultaneous linear algebraic equations. They are used widely in characterizing sequential circuits of the Moore and Mealy type, synthesis of nonlinear data converters and also in stochastic signal processing. The design procedure using signal flow graphs is also simple as compared to functional relation simulation and element simulation methods.

Ladder based Filters are used as a prototype for the transformation [1-6]. Passive ladder based filter are transformed into CFOA based circuits using Signal Flow Graph. First Kirchoff's voltage and Current Laws were applied to obtain the current equations of the ladder based prototype. The equations were then converted into Voltage form and then were used to obtain their Signal Flow Graph. CFOA based circuits for allpass and biquad filters are proposed with the help of signal flow graphs.

CFOA's are widely used in many filtering and signal processing applications. Symbol diagram of the CFOA is shown in Figure 1.

Many scientists have proposed op-amp based configuration of biquad filters. For example Jurisic et al. proposed an op-amp based configuration of biquad filter [6]. However, these circuits which are voltage mode possess a large number of disadvantages. Some of the disadvantages are:

- Gain Bandwidth product was not constant.

- The configuration was more sensitive to variations in external parameters as temperature, stray fields etc.

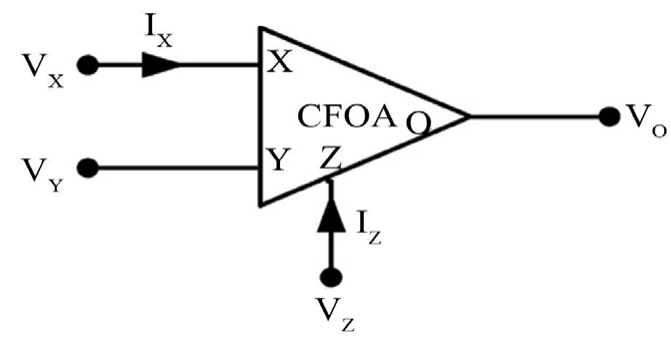

Figure 1: CFOA building block.
- Slew rate was limited.

- Configuration was having ripples in its output, hence far away from ideal output.

Many of the disadvantages were overcome by CFOA based Biquad Filter. The Circuits were nearly gain bandwidth independent. Circuits were able to produce very high slew rate which gives very high full power bandwidth. The output current has large driving capacity. Gain bandwidth is constant and variable gain amplifiers can be designed easily with CFOA based biquad filter.

Previously, Rathore and Khot [7] have designed a Low Pass and High Pass filter configuration using CFOA. They have given a systematic method of deriving CFOA based all grounded capacitor filter from Current Mode RLC prototype ladders. Sinha et al. proposed a Signal Flow Graph approach for realisation of RLC Ladder filters [8] into CFOA type filters. Referring to the Low Pass and High Pass configurations presented in his research paper $[7,8]$, we prepared two different combinations of Band Pass and Band Reject Filter presented in research paper [9]. In this paper, active realization of Allpass and Biquad filter using current feedback operational amplifier (CFOA) has been presented.

\section{Transformation of Ladder Allpass Filter into CFOA Based Allpass Filter}

All pass Filter provides phase correction and removes the interference present in magnitude response. However in practice, these responses are influenced by component variations. Since all pass functions are non minimum by definition, therefore the use of ladder based design possess many problems. The Passive ladder based all pass filter contains inductor which are bulky and takes too much space in IC

*Corresponding author: Praween K Sinha, Electronics and Communication Department, Maharaja Agrasen Institute of Technology, New Delhi, India, Tel: 01165151163; E-mail: praweenrsinha@rediffmail.com

Received December 13, 2017; Accepted April 02, 2018; Published April 09, 2018

Citation: Sinha PK, Sharma N (2018) Transformation of Ladder Based All Pass and Biquad Filters into Active All Pass and Biquad Filter using CFOA. J Electr Electron Syst 7: 253. doi: 10.4172/2332-0796.1000253

Copyright: @ 2018 Sinha PK, et al. This is an open-access article distributed under the terms of the Creative Commons Attribution License, which permits unrestricted use, distribution, and reproduction in any medium, provided the original author and source are credited. 
fabrication. Hence CFOA based all pass filter are designed which takes less space on chip. The conversion of ladder based all pass filter into CFOA based filter consists of transforming the passive filter into signal flow graph which is shown in Figure 2.

\section{Apply KVL and KCL in the circuit}

$$
\begin{aligned}
& I_{I N}=\frac{V_{I N}-V_{1}}{R_{1}} \\
& V_{1}=\frac{I_{I N}-I_{2}}{s C_{V_{2}}} \\
& I_{2}=\frac{V_{1}-V_{2}}{s L_{1}} \\
& V_{2}=\frac{I_{2}-I_{4}}{s C_{2}} \\
& I_{4}=\frac{V_{2}-V_{3}}{s L_{2}} \\
& V_{3}=\frac{\left(I_{4}-I_{6}\right)\left(s^{2} L C+1\right)}{s C} \\
& V_{0}=I_{6} R_{2} \\
& I_{6}=\frac{V_{3}-V_{0}}{s L_{3}}
\end{aligned}
$$

\section{Conversion into voltage form}

These equations are converted into voltage form to remove current element and hence we get the following equations:

$$
R I_{I N}=\frac{R\left(V_{I N}-V_{1}\right)}{R_{1}} \quad \Rightarrow V_{I N}=\frac{R\left(V_{I N}-V_{1}\right)}{R_{1}}
$$

$$
\begin{array}{ll}
V_{1}=\frac{R\left(I_{I N}-I_{2}\right)}{s C_{1} R} & \Rightarrow V_{1}=\frac{\left(V_{I N}-V_{2}\right)}{s C_{1} R} \\
R I_{2}=\frac{R\left(V_{1}-V_{2}\right)}{s L_{1}} & \Rightarrow V_{2}=\frac{R\left(V_{1}-V_{2}\right)}{s L_{1}} \\
V_{2}=\frac{R\left(I_{2}-I_{4}\right)}{s C_{1} R} & \Rightarrow V_{2}=\frac{\left(V_{2}-V_{4}\right)}{s C_{1} R} \\
R I_{4}=\frac{R\left(V_{2}-V_{3}\right)}{s L} & \Rightarrow V_{4}=\frac{R\left(V_{2}-V_{3}\right)}{s L} \\
V_{3}=\frac{R\left(I_{4}-I_{6}\right)\left(s^{2} L C+1\right)}{s C R} & \Rightarrow V_{3}=\frac{\left(V_{4}-V_{6}\right)\left(s^{2} L C+1\right)}{s C R} \\
R I_{6}=\frac{R\left(V_{3}-V_{0}\right)}{s L_{3}} & \Rightarrow V_{6}=\frac{R\left(V_{3}-V_{0}\right)}{s L_{3}} \\
V_{0}=R I_{6}=\frac{R_{2}}{R} & \Rightarrow V_{0}=V_{6}=\frac{R_{2}}{R}
\end{array}
$$

\section{Signal flow graph}

With the help of these equations we make a signal flow graph which has been shown in Figure 3.

\section{CFOA filter}

Using the signal flow graph, CFOA based allpass filter has been made as shown in Figure 4.

\section{Observations}

The passive and CFOA based ladder allpass filter circuits shown in Figures 2 and 4 are designed and evaluated in PSPICE CAPTURE using

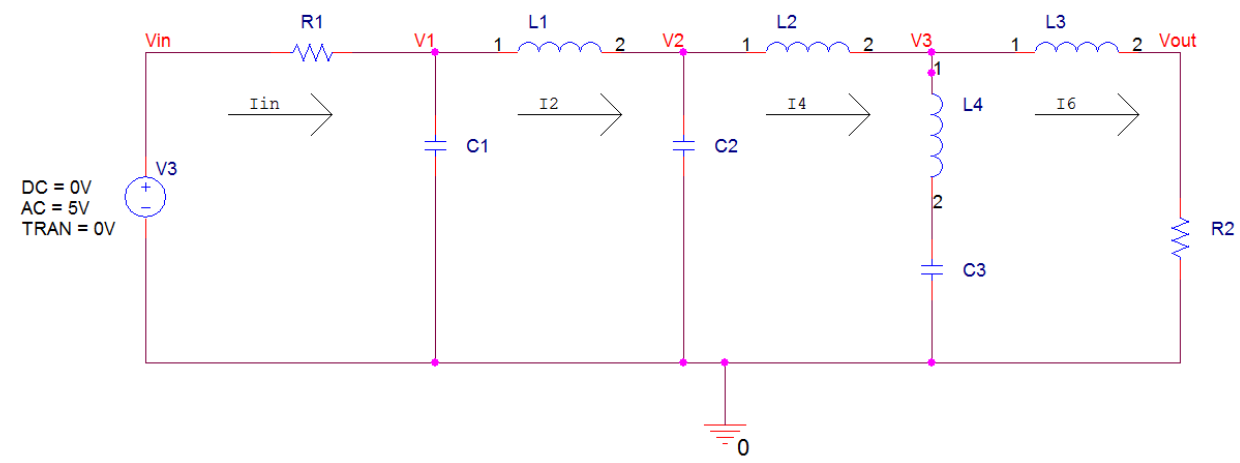

Figure 2: Ladder based all pass filter

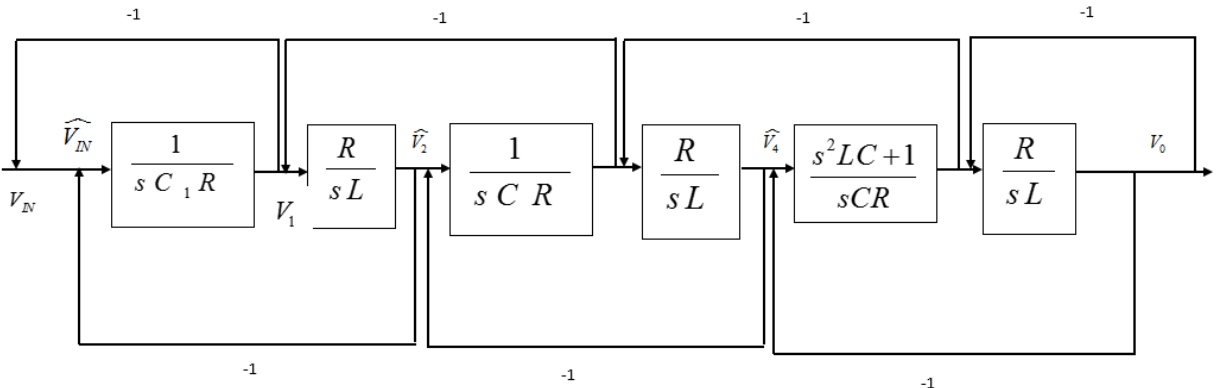

Figure 3: Signal flow graph of ladder based allpass filter. 


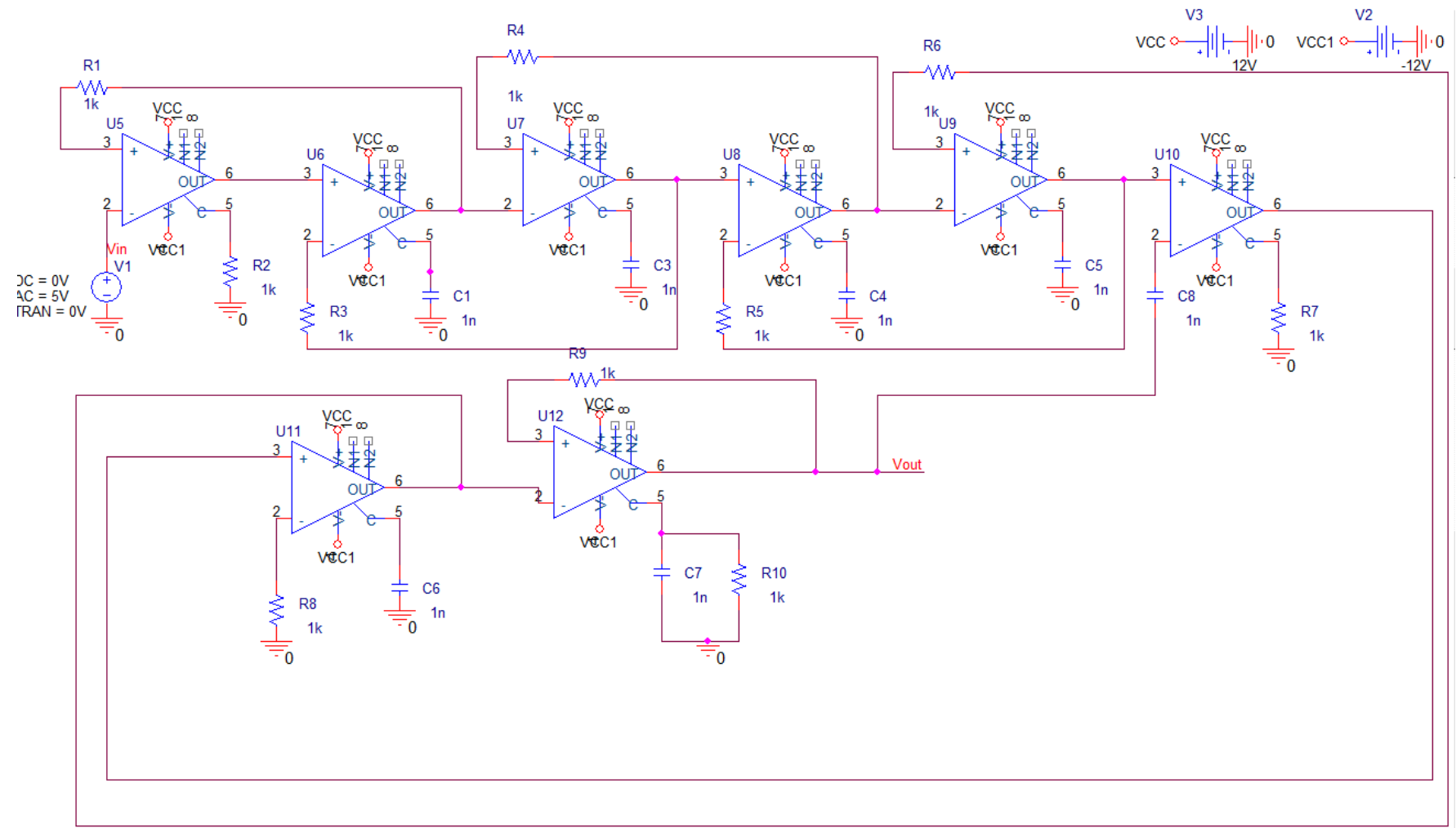

Figure 4: CFOA based Allpass filter.

conventional resistors, inductors, capacitors and AD844 IC as CFOA. Inductors are used for the passive ladder based circuit.

Now the circuit is simulated in PSPICE CAPTURE with input voltage as $5 \sin \left(2 \pi 10 \times 10^{3} \mathrm{t}\right)$ and its $\mathrm{AC}$ analysis is done.

\section{Response of CFOA based all pass filter}

CFOA allpass filter uses the AD844 IC and a power supply to drive it. The circuit is simulated in PSPICE CAPTURE with input voltage as $5 \sin \left(2 \pi 10 \times 10^{3} \mathrm{t}\right)$ and its AC analysis is done. The graphical output obtained is given below in Figure 5 .

In Figure 5, Magnitude Plot is represented by the Green colour line and the Phase Plot is represented by the Red colour line. The magnitude curve is constant and the phase varies from 0 degrees to -180 degrees.

\section{Noise response of CFOA based all pass filter}

The circuit is simulated with input voltage as $5 \sin \left(2 \pi 10 \times 10^{3} \mathrm{t}\right)$ and its noise analysis is done. The graphical result obtained is shown in Figure 6.

\section{Transformation of Ladder Based Biquad Filter Into CFOA Based Biquad Filter}

The RLC based biquad filter has been shown in Figure 7.

\section{Mathematical analysis}

After applying KVL and KCL in Figure 7, the following equations have been obtained:

$$
I_{I N}=\frac{V_{I N}-V_{1}}{R_{1}}
$$

$$
\begin{aligned}
& V_{1}=\frac{I_{I N}-I_{1}}{s C} \\
& I_{1}=\frac{\left(V_{1}-V_{2}\right)\left(s^{2} L C+1\right)}{s L} \\
& V_{2}=\frac{I_{1}-I_{2}}{s C} \\
& I_{2}=\frac{\left(V_{2}-V_{3}\right)\left(s^{2} L C+1\right)}{s L} \\
& V_{3}=\frac{I_{2}-I_{3}}{s C} \\
& I_{3}=\frac{\left(V_{3}-V_{0}\right)\left(s^{2} L C+1\right)}{s L} \\
& V_{0}=\frac{I_{3}-I_{4}}{s C} \\
& I_{4}=\frac{V_{0}}{R}
\end{aligned}
$$

Conversion into voltage form

$$
\begin{array}{lll}
R V_{I N}=\frac{R\left(V_{I N}-V_{1}\right)}{R_{1}} & \Rightarrow & V_{I N}=\frac{R\left(V_{I N}-V_{1}\right)}{R_{1}} \\
V_{1}=\frac{R\left(I_{I N}-I_{1}\right)}{s C R} & \Rightarrow & V_{1}=\frac{V_{I N}-V_{1}}{s C R} \\
R I_{1}=\left(V_{1}-V_{2}\right)\left(\frac{R}{s L}\right)\left(s^{2} L C+1\right) & \Rightarrow & V_{1}=\left(V_{1}-V_{2}\right) \frac{R\left(s^{2} L C+1\right)}{s L}
\end{array}
$$




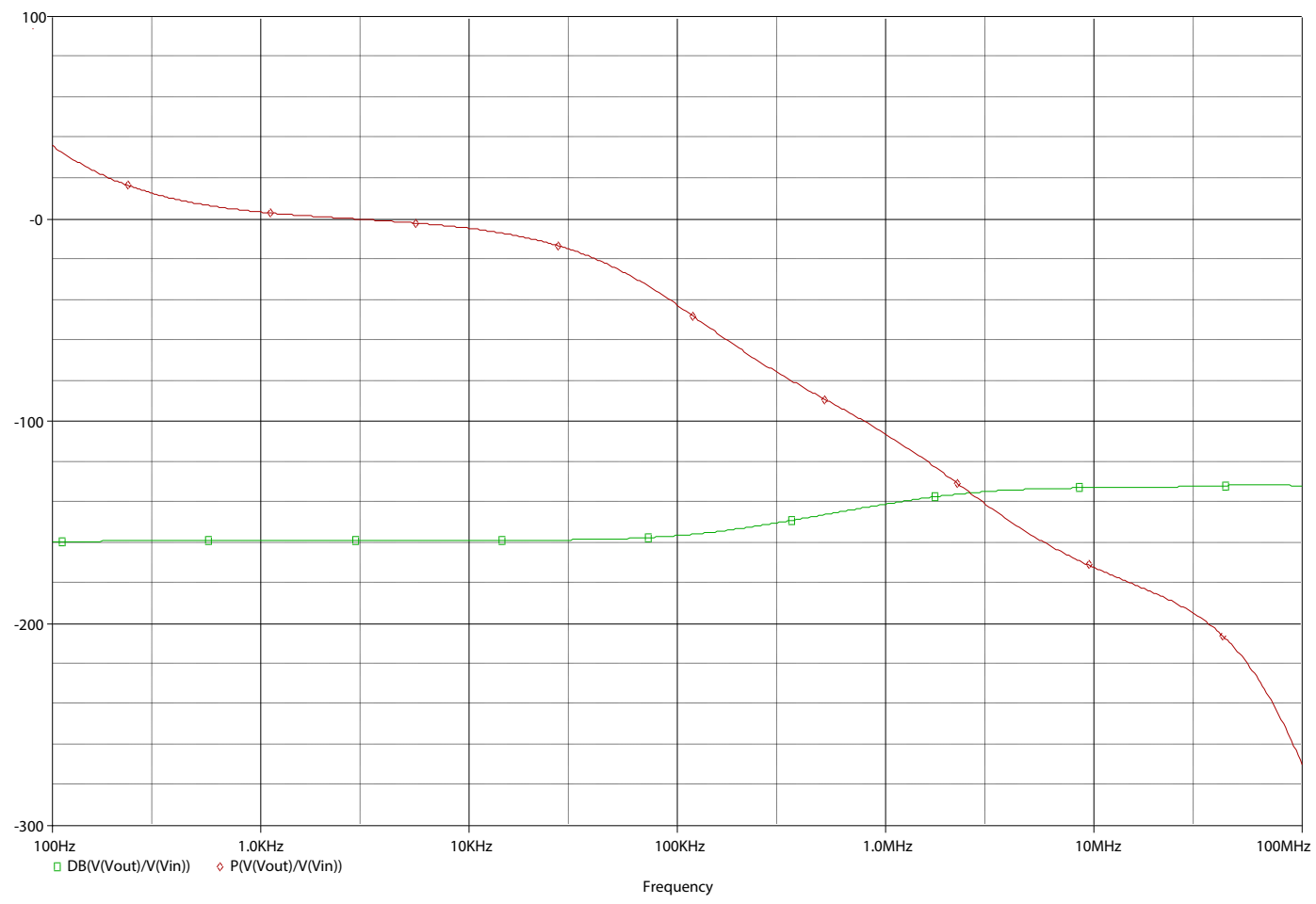

Figure 5: Response of CFOA based all pass filter.

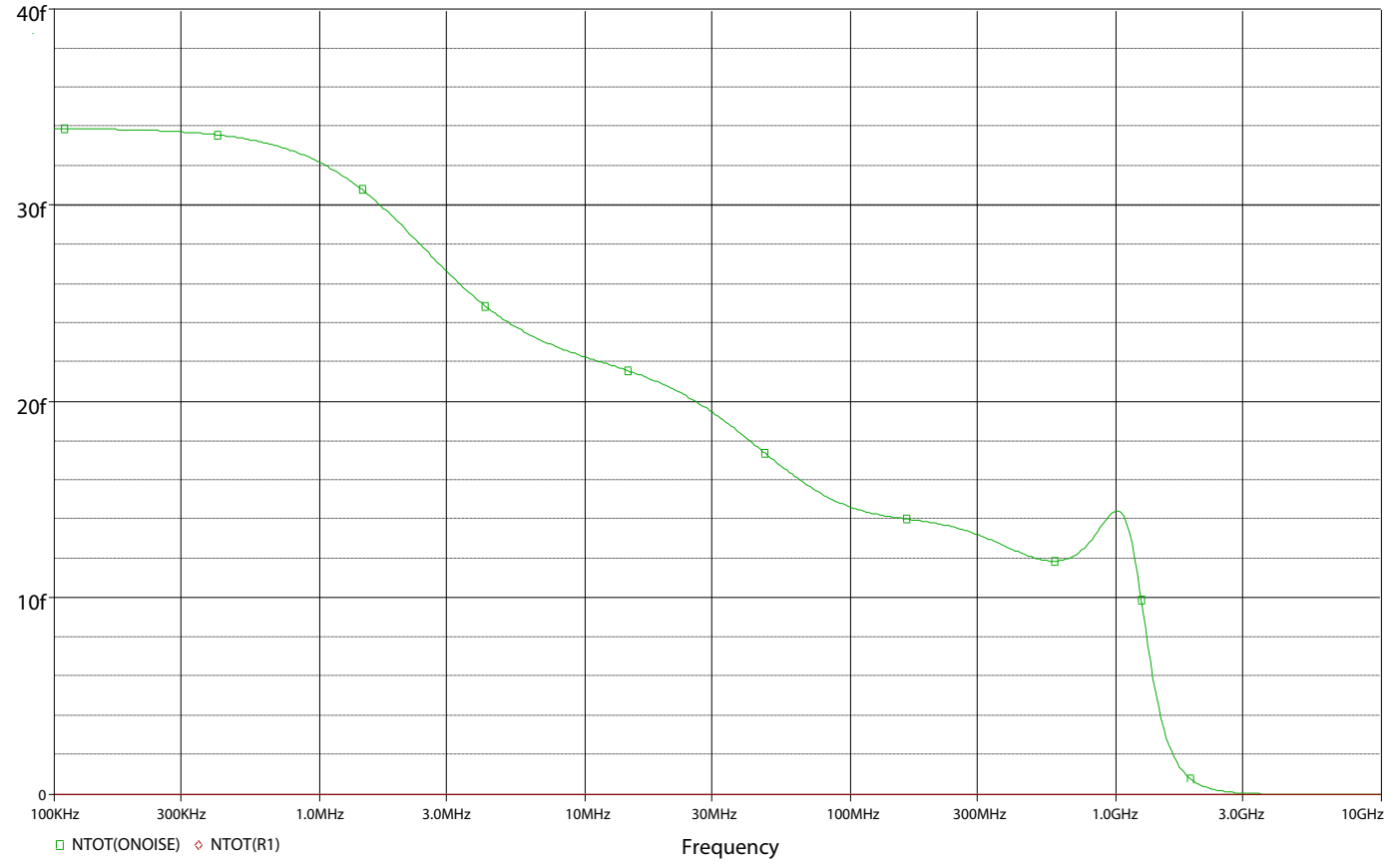

Figure 6: Noise response of CFOA based all pass filter.

$$
\begin{aligned}
& V_{2}=\frac{R\left(I_{1}-I_{2}\right)}{s C R} \quad \Rightarrow \quad V_{2}=\frac{V_{1}-V_{2}}{s C R} \\
& R I_{2}=\left(V_{2}-V_{3}\right) \frac{R\left(s^{2} L C+1\right)}{s L} \Rightarrow V_{2}=\left(V_{2}-V_{3}\right) \frac{R\left(s^{2} L C+1\right)}{s L} \\
& V_{3}=\frac{R\left(I_{2}-I_{3}\right)}{s C R} \quad \Rightarrow \quad V_{3}=\frac{V_{2}-V_{3}}{s C R} \\
& R I_{3}=\frac{V_{3}-V_{0}}{s L} R\left(s^{2} L C+1\right) \quad \Rightarrow \quad V_{3}=\frac{V_{3}-V_{0}}{s L} R\left(s^{2} L C+1\right)
\end{aligned}
$$




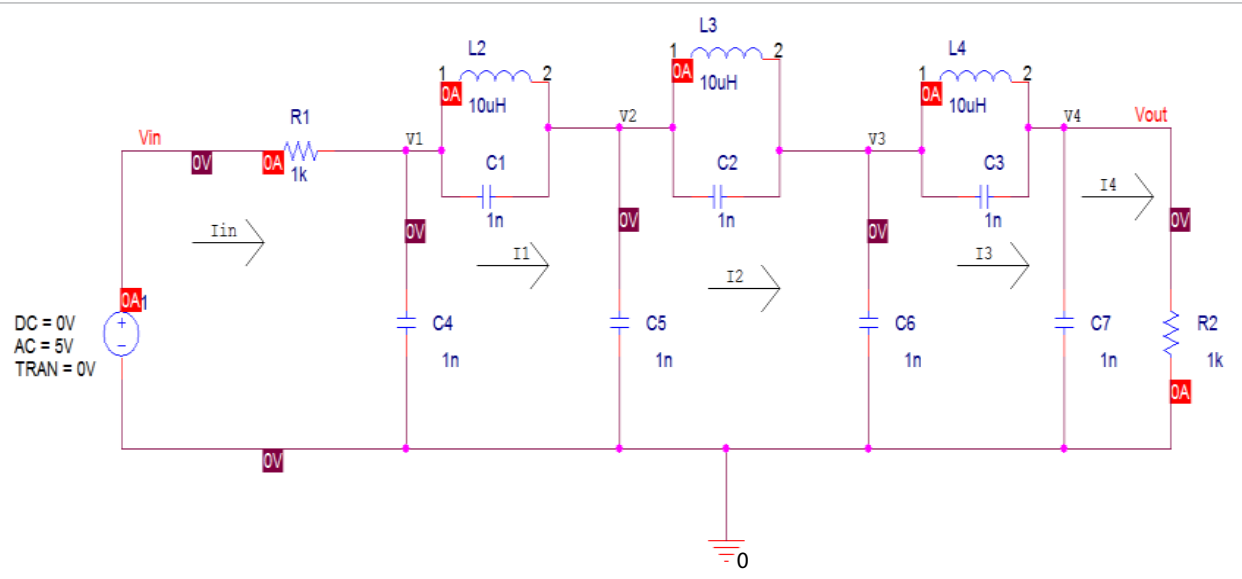

Figure 7: Ladder based biquad filter.

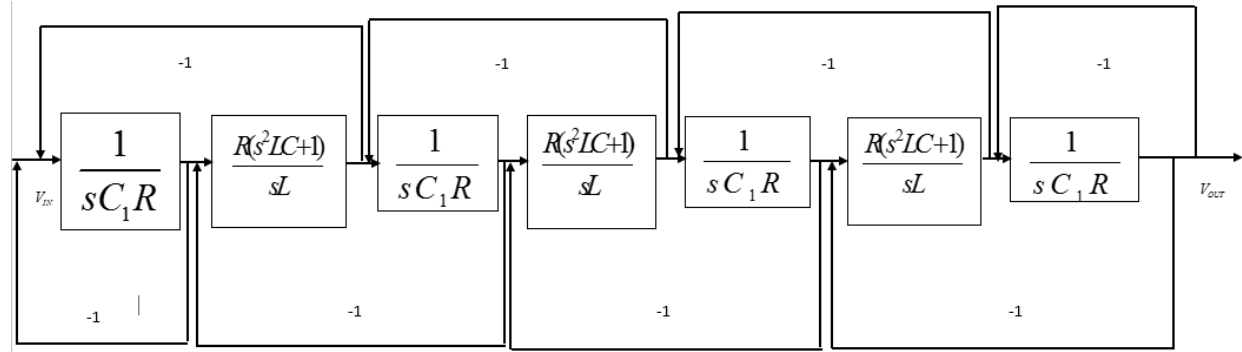

Figure 8: Signal flow graph of biquad filter (ladder based).

$$
\begin{array}{lll}
V_{0}=\frac{R\left(I_{3}-I_{4}\right)}{s C R} & \Rightarrow & V_{0}=\frac{V_{3}-V_{4}}{s C R} \\
R I_{4}=\frac{V_{0} R}{R_{A}} & \Rightarrow & V_{4}=V_{0} \frac{R}{R_{A}}
\end{array}
$$

\section{Signal flow graph}

The Signal Flow Graph of biquad filter has been shown in Figure 8.

\section{CFOA based active ladder filter}

The circuit diagram for CFOA based Biquad filter has been shown in Figure 9.

The Signal Flow Graph was analysed and the blocks were converted to CFOAdiagram. The passive and CFOA based ladder biquad filter circuits shown in Figures 7 and 9 are designed and evaluated using conventional resistors, inductors, capacitors and AD844 IC as CFOA.

\section{Frequency response (Gain vs Frequncy response)}

The circuit is simulated with input voltage as $5 \sin \left(2 \pi 10 \times 10^{3} \mathrm{t}\right)$ and its AC analysis is done. The graphical result obtained is shown in Figure 10.

\section{Noise analysis of biquad filter}

The circuit is simulated with input voltage as $5 \sin \left(2 \pi 10 \times 10^{3} \mathrm{t}\right)$ and its noise analysis is done. The graphical result obtained is shown in Figure 11.

\section{Simulation Results}

Simulation results of both the cases of All Pass and biquad Filter are as follows:

\section{Allpass filter}

All Pass Filter is simulated for $R_{1}=R_{2}=R_{3}=R_{4}=R_{5}=R_{6}=R_{7}=R_{8}=R_{9}=R$ ${ }_{10}=1 \mathrm{~K} \Omega$. Taking $C_{1}=C_{2}=C_{3}=C_{4}=C_{5}=C_{7}=C_{6}=1 \mathrm{nF}$, we have obtained the ideal frequency response.

The Magnitude response was almost constant and the Phase response varied from 0 degree to -180 degrees.

\section{Biquad filter}

A Biquad Filter is simulated for a notch frequency of $f_{n}=851.594$ $\mathrm{KHz}$, a lower cutoff frequency of $f_{l}=213.911 \mathrm{KHz}$, the lower and higher cutoff frequency for bandpass filter are $f_{l}{ }^{\prime}=1.997 \mathrm{MHz}$ and $f_{h}{ }^{\prime}=50.388 \mathrm{MHz}$.

$R_{1}=50 \Omega, R_{2}=1 K \Omega, R_{3}=500 \Omega, R_{4}=R_{8}=R_{6}=R_{7}=R_{11}=1 K \Omega$ and $R_{9}=R_{10}=$ $R_{12}=500 \Omega$. Taking $C_{1}=C_{10}=10 \mathrm{nF}$, and $C_{2}=C_{3}=C_{9}=C_{5}=C_{7}=C_{8}=C_{6}=1 \mathrm{nF}$, we have obtained the ideal frequency response.

\section{Conclusion}

In the proposed configuration implementation of ladder based allpass and biquad filter has been discussed. In the proposed configuration we convert the ladder based all pass and biquad filter using signal flow graph and implemented using CFOA based circuit. In this realisation we used grounded capacitors, CFOA, resistances by seeing the mathematical equations of signal flow graph. As the capacitors are grounded it is easy to implement in VLSI chip. The author already discussed the implementation of low pass, high pass, band pass and band reject ladder based filter and its conversion into CFOA based circuit. In this paper allpass and biquad configuration has been discussed and verified by Pspice simulation. 


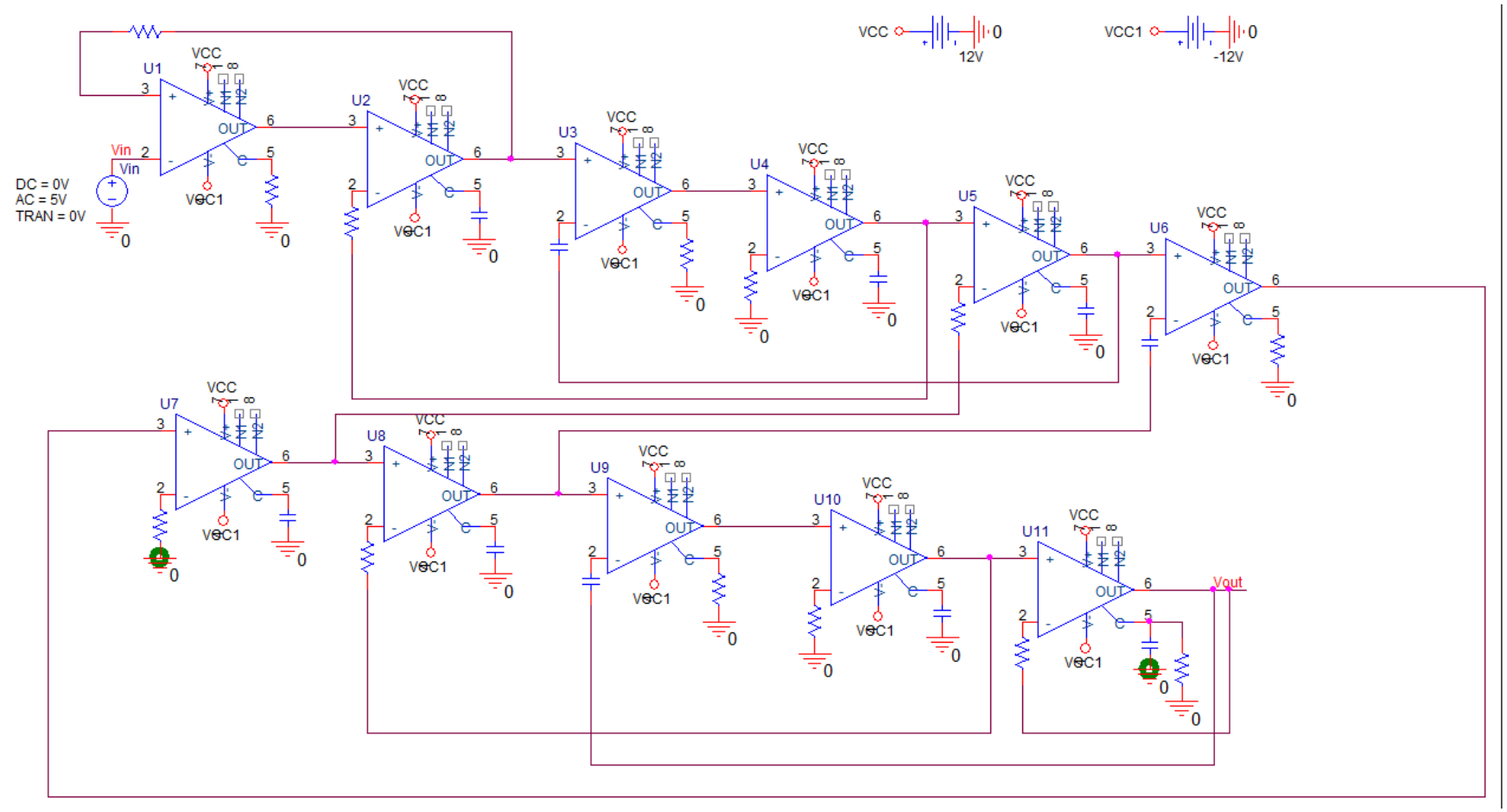

Figure 9: CFOA based biquad filter.

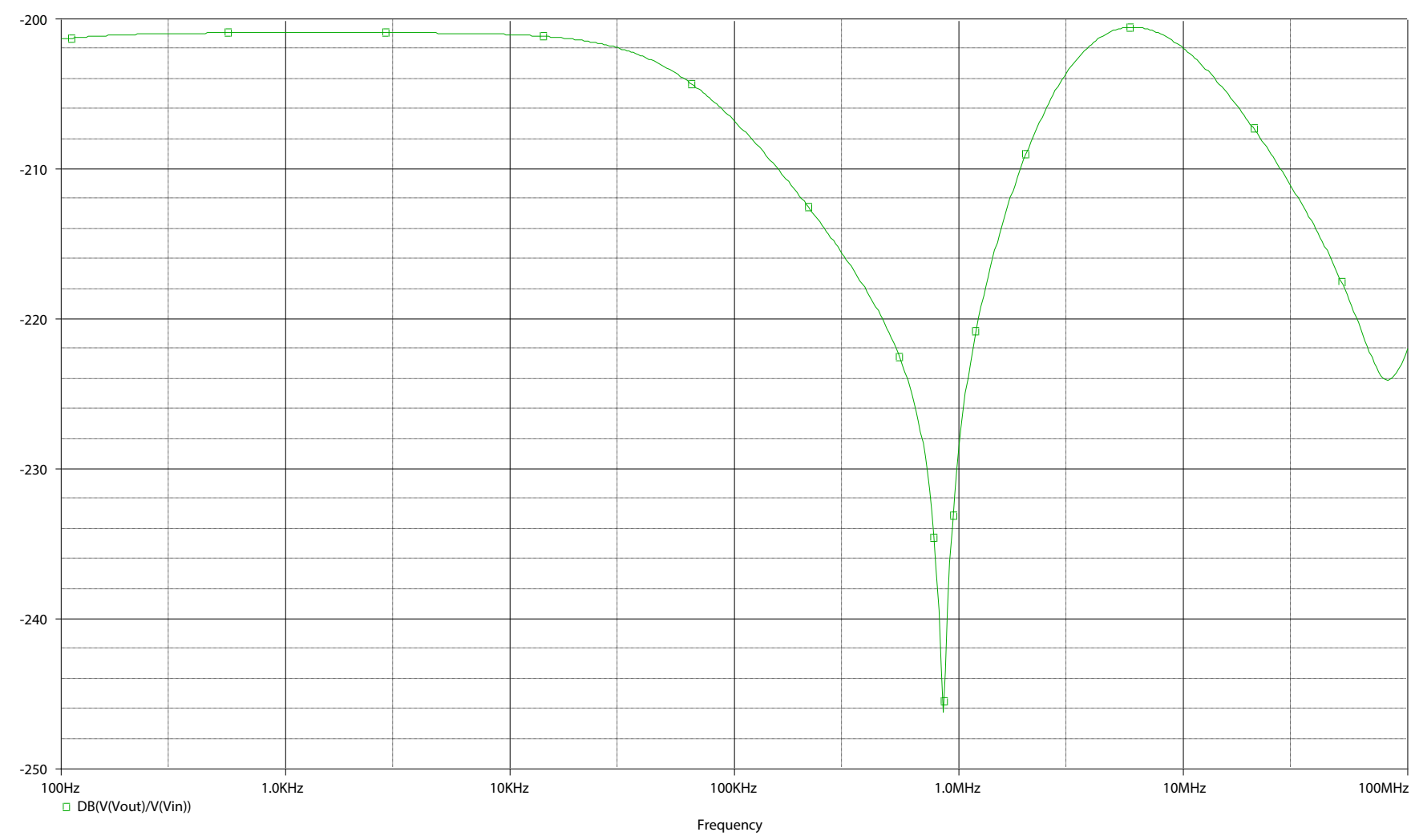

Figure 10: Response of CFOA based biquad filter. 
Citation: Sinha PK, Sharma N (2018) Transformation of Ladder Based All Pass and Biquad Filters into Active All Pass and Biquad Filter using CFOA. J Electr Electron Syst 7: 253. doi: 10.4172/2332-0796.1000253

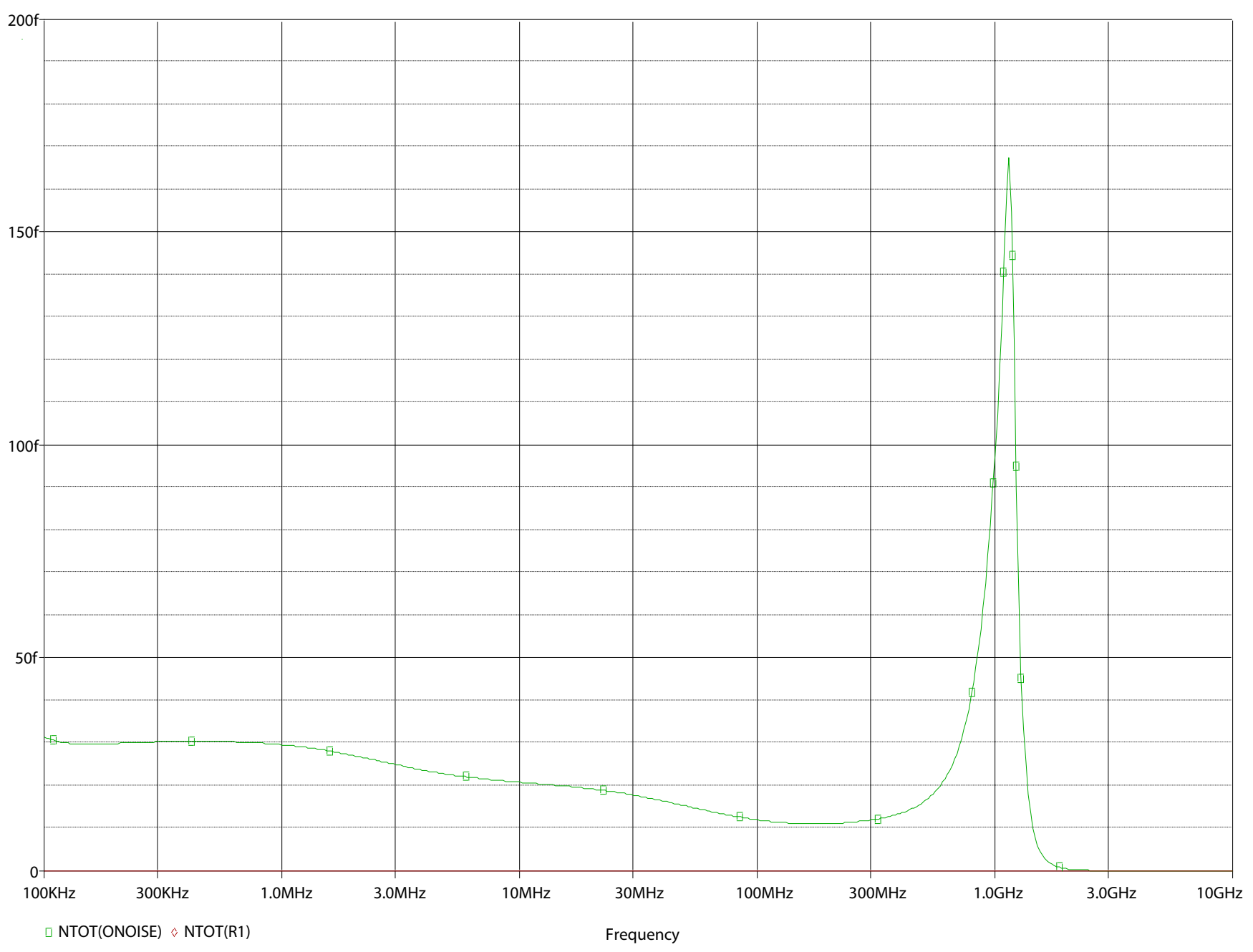

Figure 11: Noise response of CFOA based biquad filter.

\begin{tabular}{|l|l|l|}
\hline Filter & Active Elements & External Resistors \\
\hline All pass filter & $8 \mathrm{CFOA}$ & 10 Grounded \\
\hline Biquad filter & $11 \mathrm{CFOA}$ & 11 Grounded \\
& & \\
& & \\
& & \\
& & \\
\end{tabular}

\begin{tabular}{|l|l|l|}
\hline External Capacitors & Frequency & \\
\hline 6 Grounded & Phase varying from 0 degrees to -180 degrees \\
\hline & Low pass filter & $f_{1}=213.911 \mathrm{KHz}$ \\
\hline & Notch filter & $f_{\mathrm{n}}=851.594 \mathrm{KHz}$ \\
\hline \multirow{3}{*}{ Band pass filter } & $f_{l}{ }^{\prime}=1.997 \mathrm{MHz}$ \\
\hline & $f_{h}{ }^{\prime}=50.388 \mathrm{MHz}$ \\
\hline
\end{tabular}

Table 1: Results of proposed realisation.

The summary of the components used the various frequencies and the bandwidth of Allpass and biquad filter used in our configuration has been illustrated in Table 1 .

\section{References}

1. Lee SS, Zele RH, Allstot DJ, Liang G (1993) CMOS Continuous-Time CurrentMode Filters for High-Frequency Applications. IEEE J Solid-State Circuits 28: 323-329.

2. Ramirez-Angulo J, Sanchez-Sinencio E, Robinson M (1992) Current-Mode Continuous Time Filters: Two Design Approaches. IEEE Trans Circuits Syst 39: $337-341$

3. Hughes BJ, Bird CN, Macbeth Cl (1989) Switched Current-A New Technique for Analog Sample-Data Signal Processing. IEEE Int Symp Circuits Syst Proc, Portland 1989: 1584-1587.

4. Wu J, El-Masry E (1996) Current-Mode Ladder Filters Using Multiple Output Current Conveyors. IEE Proceedings-Circuits, Devices and Systems 143: 218222.

5. Wu J, El-Masry E (1997) A New Approach of Design of Current-Mode Filters. IEEE Int Symp Circuits Syst 1: 317-320.

6. Jurisic D, Mijat N, Moschytz GS (2005) Low-Sensitivity Current-Mode ActiveRC Filters Using Impedance Tapering. IEEE Int Symp Circuits Syst 4: 33033306.

7. Rathore TS, Khot UP (2008) CFA-Based Grounded-Capacitor Operational Simulation of Ladder Filters. Int J Circ Theor App 36: 697-716. 
Citation: Sinha PK, Sharma N (2018) Transformation of Ladder Based All Pass and Biquad Filters into Active All Pass and Biquad Filter using CFOA. J Electr Electron Syst 7: 253. doi: 10.4172/2332-0796.1000253

Page 8 of 8

8. Sinha PK, Saini A, Kumar P, Mishra S (2014) CFOA Based Low Pass and High Pass Ladder Filter-A New Configuration. Circuits and Systems 5: 51319.
9. Sinha PK, Sharma N, Agarwal S, Saha S (2016) CFOA Based Band Pass and Band Stop Ladder Filter-A New Configuration. Circuits and Systems 7: 62907. 